\title{
A Study for Building Smart Home Environment Based on Modular Equipment Design Concept
}

\author{
Seokjin Kim \\ Department of Industrial Art, Faculty of System Design, Tokyo Metropolitan University, Tokyo, Japan \\ Email: kimusj@sd.tmu.ac.jp
}

Received 2 February 2015; accepted 23 April 2015; published 24 April 2015

Copyright (C) 2015 by author and Scientific Research Publishing Inc. This work is licensed under the Creative Commons Attribution-NonCommercial 4.0 International License (CC-BY-NC). http://creativecommons.org/licenses/by-nc/4.0/ cc) (i) (8) Open Access

\begin{abstract}
In this paper, we propose a modular equipment design concept for building smart-home environment, which can deal with the problems such as life expectancy and complicated cable networks. Recently, the advancement of digital technology in residential networking and connections (i.e., internet cables, power cables, and network cables) has made a positive alteration to the interspatial relationship between appliances. Moreover, the interaction and communication between user and application has understood progressive changes. In future home environment, the living room is a place that has become a haven for the family and a place for entertainment as well. The downside of the new technologies is the life expectancy of the product, which is affected by a simple change to the development of digital technology.
\end{abstract}

\section{Keywords}

Modular Design, Home Electronics, Network, Home Environment

\section{Introduction}

As digital products have the attribute of being function-centered, they offer the possibility of fusing furniture and space within the home (Dresselhaus, 2004; Kim, 2007; Kim, 2008). These new forms of digital-conversion home electronics may greatly change our daily lives and home environments. In the future, the home environment equipped with a digital network will not simply be a place for the family to relax, but is also expected to play a role as the final destination for the various data produced by information society, and as a place for the consumption and production of information. In other words, at the same time as being a place to relax, it will be 
a place for work, a place for enjoyment, and a place for learning (Newman, 1998; Weiser, 1991). Therefore, a new paradigm is required alongside the changes to products. Since the digital revolution, in conjunction with the development of information-processing equipment and home electronics, various technologies have entered into our living spaces and gradually become intelligent (Munro, 1991; Munn, 1989). At the same time, new relationships need to be established between home electronics and home spaces. These changes are taking place against the backdrop of the emergence of products that blur the distinction between home electronics and furniture (Wimmer, 2001). This research looks directly at the roles of digital technology in home electronics and furniture, and proposes a new product image for the future from the fusion of home electronics, furniture, and spaces that are created from the interactions between them. The aim is to create a new conception of the home electronics environment from digital technologies and culture.

\section{Background to the Research}

\subsection{The Relationship between Behavior in the Living Space and Furniture}

If we exclude going to bed and housework, the majority of activities in the home space occur centered in the living room. Many of the actions in our daily lives, such as acquiring information, communication, and entertainment are accomplished in the living room. Therefore, there are expectations for the rational utilization of furniture within it; specifically, for the fusion and connection of home electronics and furniture. Within this, the technology that will be used in the homes of the future and the module home electronics that are constantly advancing need to be developed into a form that can continuously respond to changes in technologies and lifestyles. In the living space shown in Figure 1, the appliances that are used, which are centered on the members of the family's acquisition of information, communication, and entertainment duplicate each other but are also different in terms of shape, size, and standards. This is the current situation, in which they are all jumbled together within a narrow space. Therefore, there is a need to propose a new integrated form of home electronics through their physical and technological fusion, the modularization of size, and moreover, the application of a new interface for composite devices (Figure 2).

Speaker

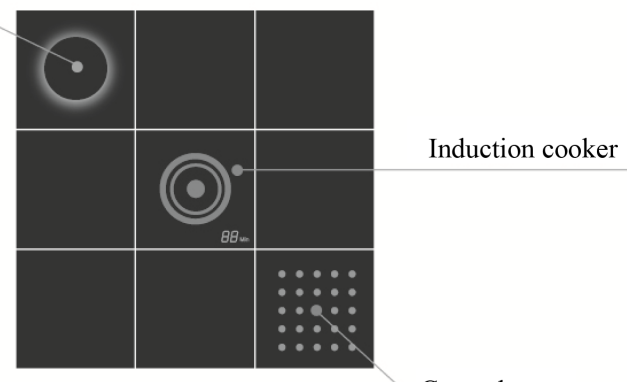

Controler

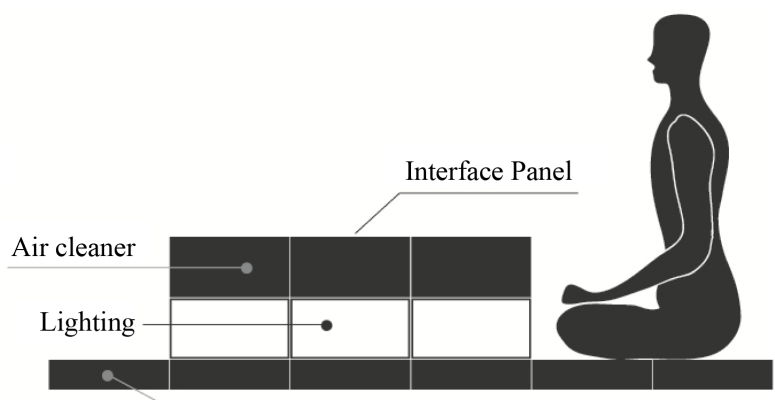

Network Module

Figure 1. The module structure.
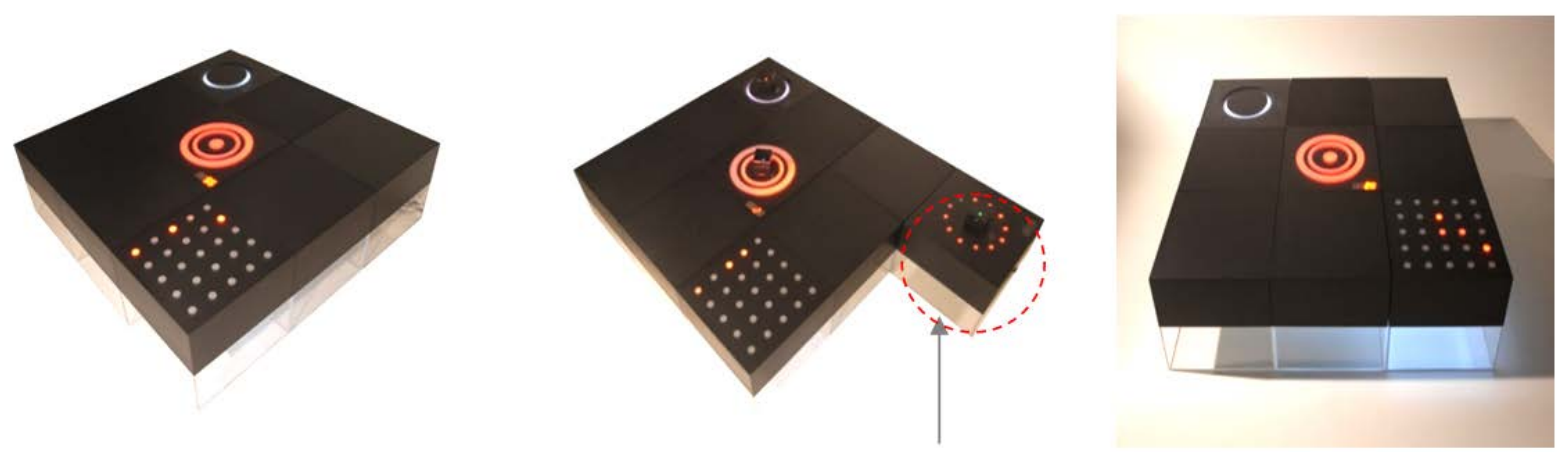

Home Server Modulle

Figure 2. The module combination for a communications table. 


\subsection{Changes to the Living Space through the Fusion of Home Electronics and Furniture}

Figure 3 (left) shows a pattern frequently seen in the layouts of existing living spaces, with the table and couch placed at the center of the living room and home electronics by the walls. A constant distance is maintained from the couch to the TV, and the various related devices are kept on the AV rack centered on the TV. In this living room, the members of the family sit on the sofa orientated in a single direction, and basically use the remote controls to operate the equipment. The fusion of home electronics and furniture greatly changes this arrangement and spatial configuration. The members of the family gather centered on the living space and come to be able to converse and enjoy entertainment while facing each other (Figure 3 (right)). In addition, when operating home electronics, they are able to easily use a wide range of functions through an intuitive interface that utilizes their five senses.

\subsection{Current Problems}

In the environment surrounding the TV in the living room, there are at least two or three types of electronic devices, mainly on the AV rack, and in extreme cases there might be 10 or more. But a power supply that is suitable for this number of devices is required. Also, currently, an indispensable element of home electronic devices is that they are constructed to mutually connect with each other via a network. Consequently, when we connect the Internet, the related devices, and the network line, we end up with a complex network of cables within the living space, greater than we imagined (Figure 4).

\section{Design Concept}

The modularization of functions makes possible home electronics from the selection and combination of digital units suitable for each individual's lifestyle, while the freedom to determine layout through modularization realizes home electronics that demonstrate "individuality within rules". This is the design concept of "sustainable home electronics", which can partially upgrade rapidly changing network technologies. Based on this new form of interaction generated from new definitions of the relationships between people and objects, and also between the objects themselves, a new concept for home electronics that can fuse culture and technology is here proposed (Figure 5).

\subsection{Module Design Keywords (Figure 6)}

- Discovery of elements to fuse home electronics, furniture, and spaces

- Development of modules that can incorporate technology
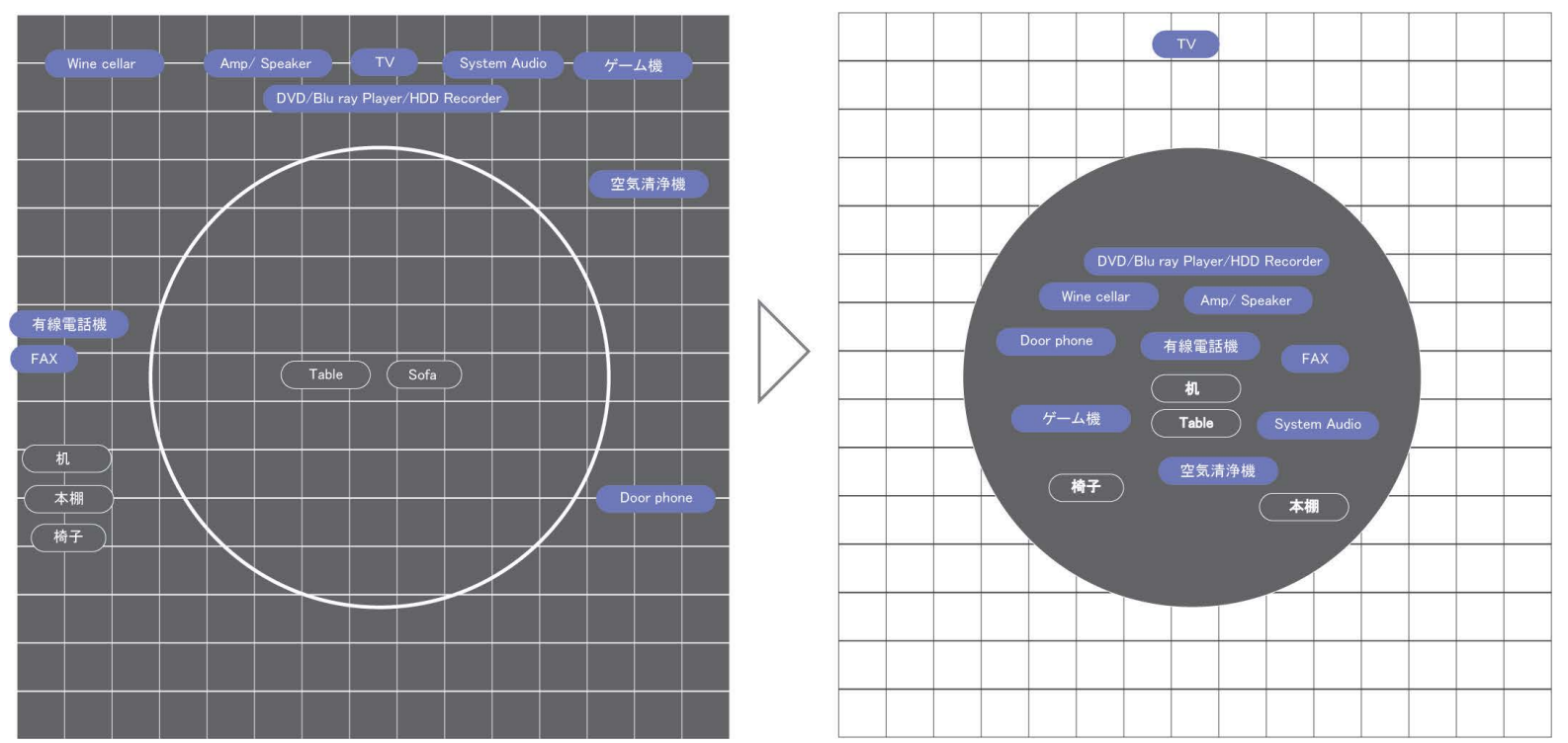

Figure 3. The existing layout of home electronics and furniture. The new layout following their fusion. 

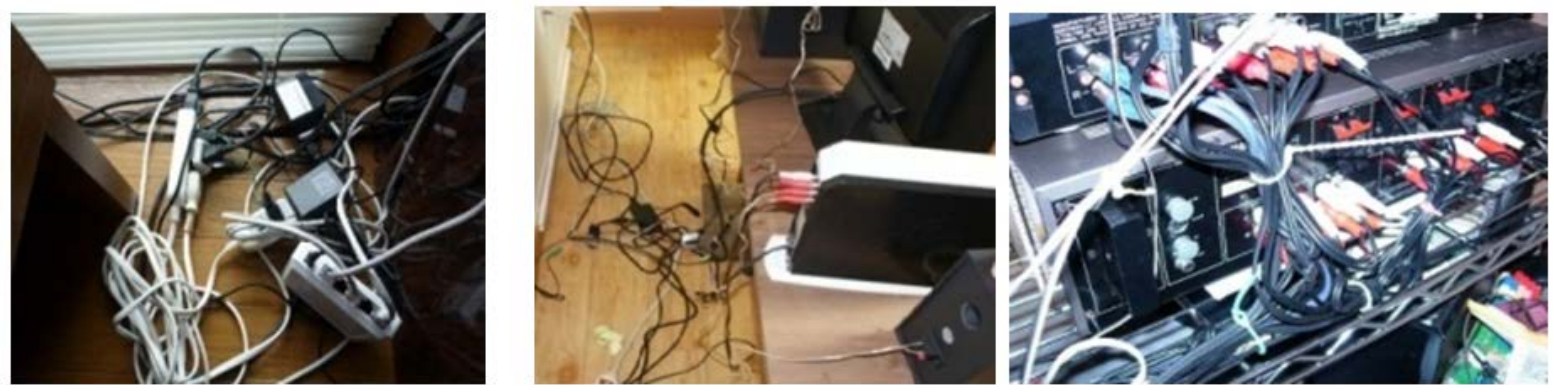

Figure 4. Power cable, internet cable, network cables within the home.
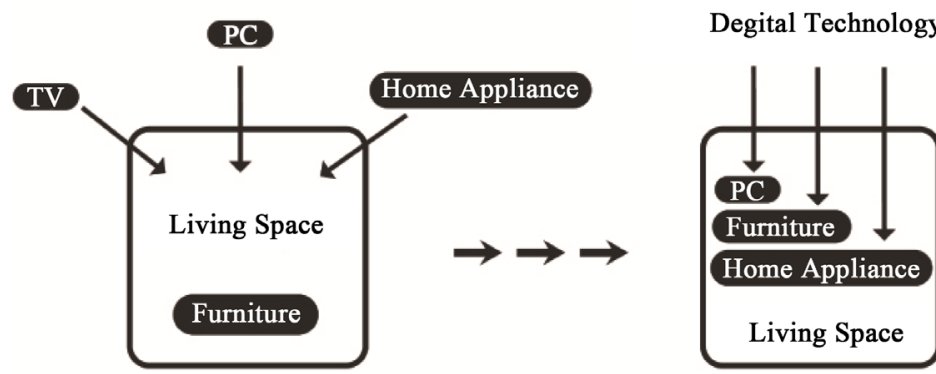

Convergence \&

Network

Figure 5. The relationships between home electronics, furniture, and spaces through digitalization.
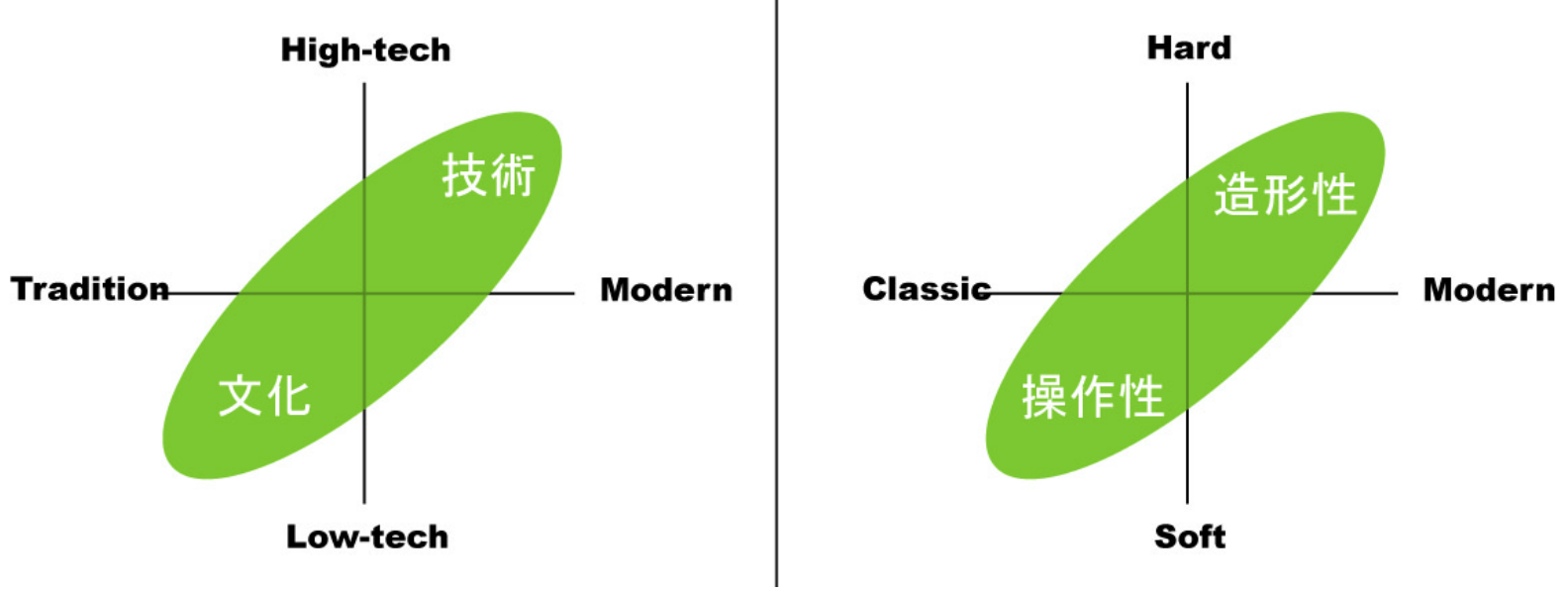

Figure 6. The relationship between design technologies and culture, and the relationship between form and operationality.

- Extension of product lifespans by the partial replacement of modules

- Proposal for a structure that can continuously respond to changes

- Proposal for a lifestyle with a free-layout structure

\subsection{The Module's Design}

To connect digital home electronics with spaces, it is necessary to establish and then share elements such as predetermined design regulations and module size.

\subsubsection{Design Principle 1 (Basic Module)}

For the basic module size $(300 \times 300 \times 150)$, the focus is currently being placed on improving its feasibility further, for example its assembly and disassembly, by setting basic units of size for the distributed architectural components. In addition, focusing on the module, the greatest emphasis is being placed on a design that increases the freedom to adapt the layout of the furniture and space. The functional structure of the module was conceived 
to achieve composite multi-functions by installing one function in one module box, and then combining the modules. The types of installed functions are divided into categories centered on the genres of existing equipment, including AV equipment, computer-related products, and telecommunications equipment. In addition, the aim is for a module design that can be divided into a spatial element and a furniture element in accordance with a physical scale that can accommodate these devices.

\subsubsection{Design Principle 2 (Coordination with the Furniture and the Space)}

Basically, one module is equipped with one function. The module can exist as an independent unit, but it is also possible to increase its functions through connecting it with modules with other functions. Also, by connecting the modules, it is possible to not only expand the product's functions but, in addition to this, its physical function as furniture. In other words, while functioning as home electronics, they can also be used as furniture through the various combinations of modules. To take it one step farther, it is possible to apply this to walls, ceilings, pillars, floors, and the other elements that form the building. In this way, it is considered that by not restricting a module with one function to simply being physical hardware but instead expanding it into a wider space (furniture and the building), the module is able to continuously respond to changes to the environment.

\subsection{The Module Structure}

\subsubsection{Definition of the Function Unit}

In the product structure, it incorporates the functions and is responsible for direct interface with the user. It is necessary to visually express the functions of the product, and the operation of the functions is accomplished by an internal touch sensor on the surface. Changes to the internal digital source can be upgraded via the Internet. It also functions as a display by using materials that transmit light. It must be heat resistance and durable (Figure 7).

\subsubsection{Definition of the Sub Unit}

The sub unit plays the role of physically supporting the main unit's digital functions. It provides a human scale adapted to the user by transforming the layout, and it exists as analog-type furniture connecting the product to the space. One unit can be physically attached either to the top or bottom to create a stack. It utilizes natural materials, such as wood, and new materials that transmit light, to produce lighting and other effects.

\subsection{The Network Space Module}

\subsubsection{The Wall Module System}

In order to connect the home network basic equipment and the digital modular home electronics, it is necessary to use power cables, Internet cables, and moreover network cables that interconnect devices. These cables are usually pulled into the changeable walls that compose the space. In particular, as the network cable is the part that is in direct contact with the home-electronics product, it can be given a visually clean impression by building it into the wall and concealing the cables. It is highly possible that, in a few years, technology will become wireless.

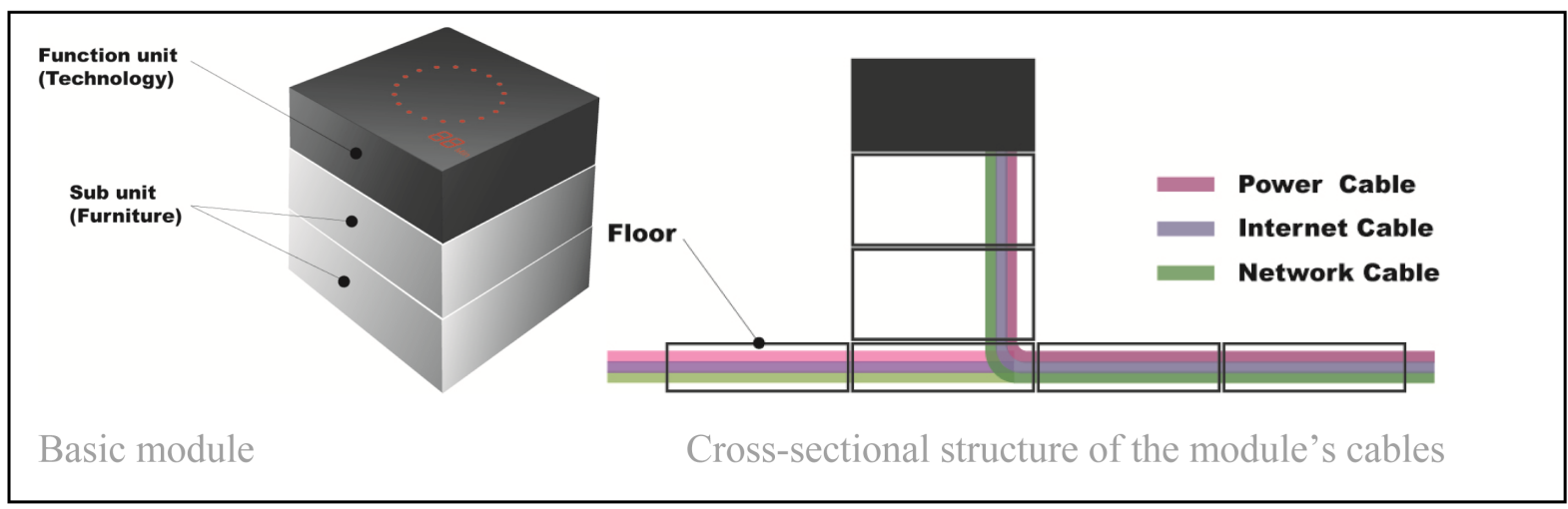

Figure 7. Basic module and cross-sectional structure of the module’s cables. 


\subsubsection{The Floor Module System}

The horizontal space is broadly divided into the ceiling and the floor. The ceiling is equipped with the air-conditioning equipment, and includes lighting. As practically all of the home electronics can be set up on the floor, power cables, Internet cables, and network cables are needed. By creating a module system, the signal from each cable is supplied logically to the digital units or home electronics on the floor (Figure 8).

\subsection{The Interface}

The fusion of home electronics (digital area) and furniture (analog area) creates a new interface. In particular, the part that people are directly in contact with is operated using their five senses, and so extra modular elements for the operations system can be eliminated, facilitating more freedom for the module layout. As shown in Figure 9, even if the module's physical capacity changes, such as by changing its height to match the human scale, the digital area (operations system) can be operated intuitively by a layout centered on the radius of action.

\section{Communication Table}

Figure 10 shows the functional structure. Individual information and cooking, simple cooking equipment, and

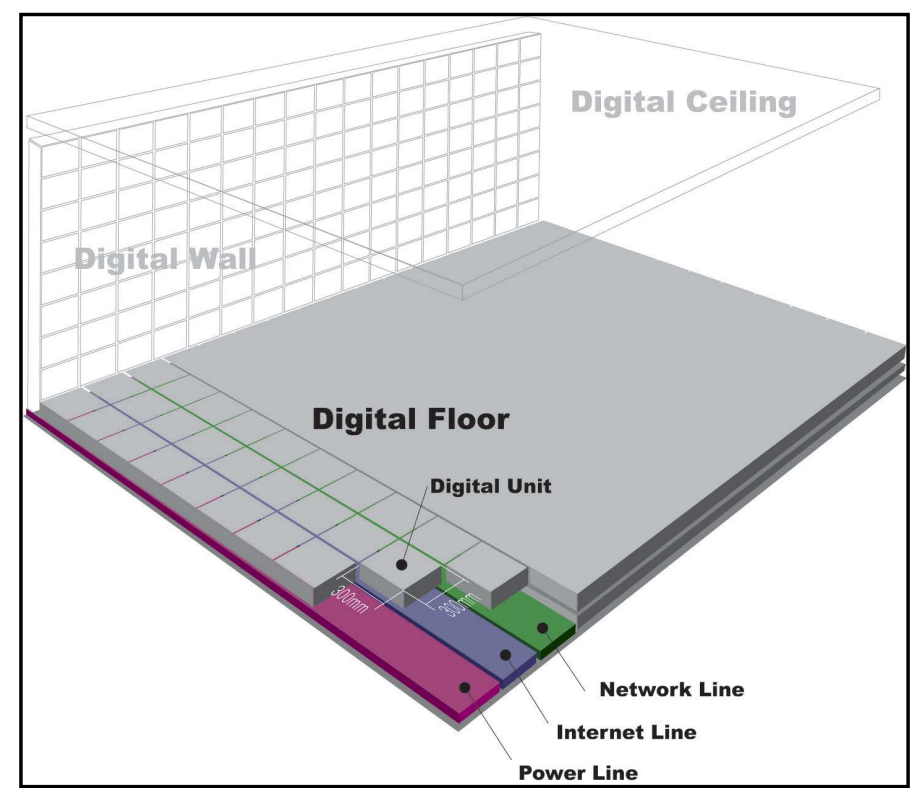

Figure 8. The plan of the floor module system.

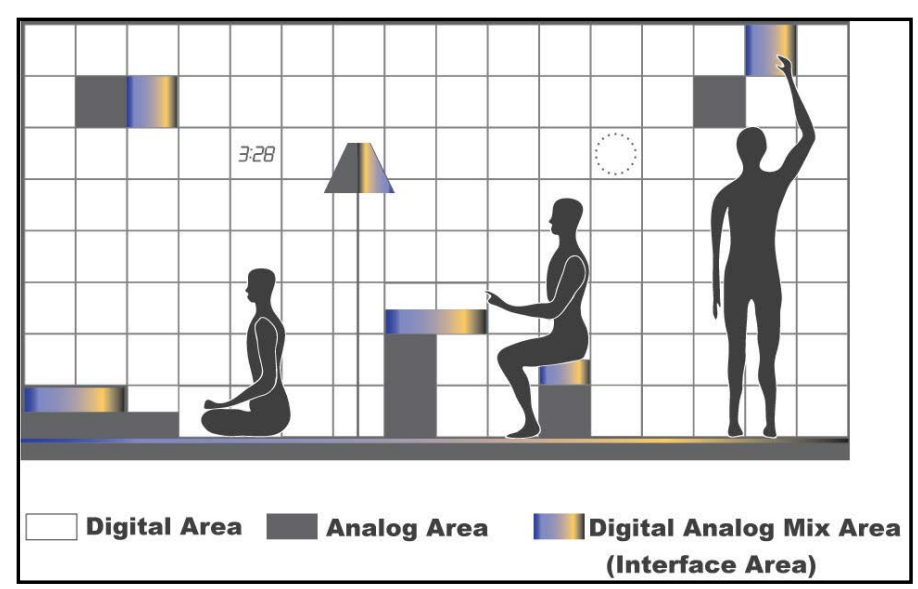

Figure 9. Elevation of the unites in the module space. 


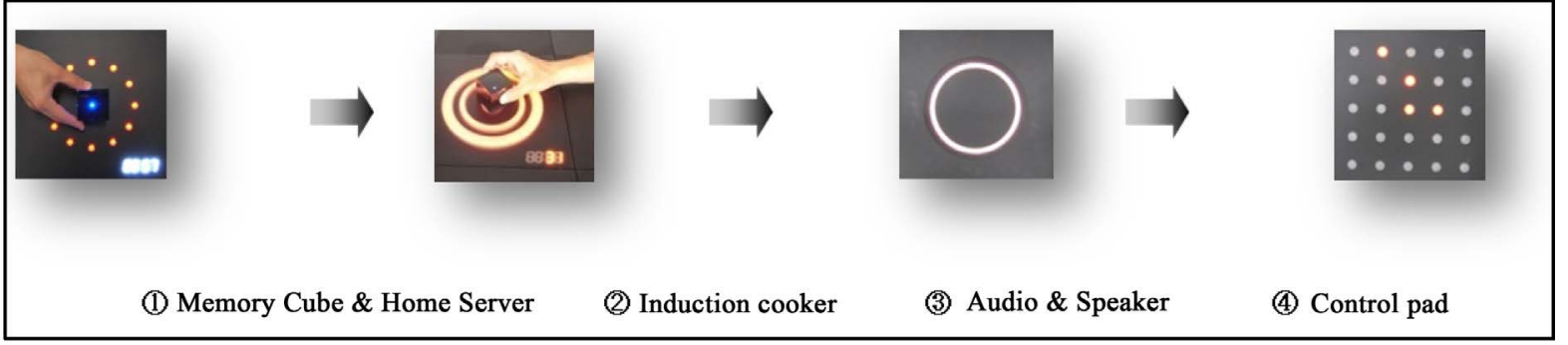

Figure 10. Communication table.

playing music, according to the genre, cooking data, music download audio data, such as music data transmit audio data fluctuate the lighting control.

\section{Conclusions and Issues for the Future}

Within living spaces made intelligent by digital technology, we must extend concepts in order to create sustainable products that exist alongside people. Also, by introducing the medium of furniture, it becomes possible to construct new spaces or new lifestyles. The "communications table" is only one example of module home electronics described by the author. But looking toward the digital network society of the future, we can expect the concepts we have held for home electronics up to the present time to be greatly transformed. In terms of issues for the future, in order to more smoothly connect home electronics to spaces, research is required to rationalize the modules through their connective structure and to also rationalize the interface so it is able to transmit digital functions without any sense of incompatibility. In conjunction with this research, work is needed to discover and develop new materials.

\section{References}

Dresselhaus, B. (2004). Design Innovation 1.0. Portland: Dresselhaus Design Group.

Kim, M. (2008). Understanding of Ubiquitous. Seoul: Ihan.

Kim, N. (2007). A Study on the Modula Coordination for the Smart Home. Seoul.

Munn, R. E. (1989). Towards Sustainable Development: An Environmental Perspective. Economy and Ecology: Towards Sustainable Development. New York.

Munro, D. A. (1995). Sustainability: Rhetoric or Reality? In Trzyna, \& Thaddeus (Eds.). California: Center for the Environment and Public Policy.

Newman, P. (1998). Sustainability and Cities. Washington: Island Press.

Weiser, M. (1991). The Computer for the 21st Century. New York: Scientific American Press.

Wimmer, W. (2001). ECO Design Pilot. Massachusetts: Kluwer Academic Publishers. 